\title{
LAZER UNIVERSITÁRIO: ESTUDOS RECENTES SOBRE PRÁTICAS QUE ENCORAJAM O BEM-ESTAR SOCIEDUCACIONAL DE JOVENS ESTUDANTES
}

Patricio Cuenca Nicolás
Profesor Asociado. Universidad de Murcia patricio.cuenca@um.es

Para citar este artículo puede utilizar el siguiente formato:

Patricio Cuenca Nicolás: "Lazer universitário: estudos recentes sobre práticas que encorajam o bemestar socieducacional de jovens estudantes", Revista Caribeña de Ciencias Sociales (Especial noviembre 2021, pp. 62-73) En línea:

https://doi.org/10.51896/caribe/HRES7432

\section{RESUMO}

O lazer como fenômeno social e experiência pessoal adquiriu, nos últimos anos, uma importância que não adquiria desde a antiguidade. Vivemos uma transformação relevante de atitudes em relação ao lazer, cuja consequência é o aumento da importância desta área da vivência social no bem-estar, na satisfação e na qualidade de vida em geral. Também tem induzido, entre pesquisadores e universidades, um aumento do interesse em explicar e conhecer esse fenômeno, que se acredita ser o elo que faltava para a compreensão do comportamento e da sociedade atual. Este artigo tem como objetivo explorar as pesquisas realizadas que relacionam o lazer e o tempo livre com a vida do estudante universitário. Essas investigações evidenciam a importância do lazer entendido como práticas que buscam relaxamento, descanso e desligamento das rotinas do dia-a-dia e, por outro lado, todas aquelas atividades que têm a ver com as práticas de convivência e / ou vivências. relacionadas aos cuidados físicos de cada indivíduo. Além disso, os estudos discutidos no artigo destacam os fatores inclusivos que ocorrem entre os estudantes universitários e aqueles relacionados ao bem-estar socioeducativo, o florescimento de valores que melhoram a convivência social e a melhoria da qualidade de vida: práticas culturais, formativas ou experiências relacionadas ao turismo.

Palavras-chave: lazer universitário, qualidade de vida, práticas culturais, vida do jovem estudante e bem-estar socioeducativo.

\section{UNIVERSITY LEISURE: RECENT STUDIES ON PRACTICES THAT ENCOURAGE THE SOCIEDUCATIONAL WELFARE OF YOUNG STUDENTS}

\section{ABSTRACT}

Leisure as a social phenomenon and personal experience has achieved, in recent years, an importance that it had not obtained since ancient times. We are experiencing a relevant transformation 
of attitudes towards leisure, the consequence of which is the increasing importance of this area of social experience in well-being, satisfaction, and quality of life in general. It has also induced, among researchers and universities, an increase in interest in explaining and learning about this phenomenon, which is believed to be the missing link in the understanding of behavior and current society. This article aims to explore the research carried out that relates leisure and free time to university student life. These investigations highlight the importance of leisure understood as practices that seek relaxation, rest and disconnection from day-to-day routines and, on the other hand, all those activities that have to do with the practices of socializing experiences and / or that they are related to the physical care of each individual. In addition, the studies discussed in the article highlight the inclusive factors that occur among university students and those related to socio-educational wellbeing, the flowering of values that improve social coexistence and the improvement of quality of life: cultural practices, formative or experiences related to tourism.

Keywords: university leisure, quality of life, cultural practices, youth student life and socio-educational well-being.

\section{INTRODUCCIÓN}

O trabalho de investigação que se reflecte a seguir é uma revisão de vários estudos e documentos que têm como objetivo: analisar e explorar as variáveis e fatores derivados do lazer universitário juvenil, tendo em conta a revisão sistemática da bibliografia. Consequentemente, são extraídas várias experiências de vida universitária e como elas atendem à melhoria da qualidade de vida e do bem-estar social de cada aluno e às atividades mais marcantes que surgem entre os jovens. Atualmente, estão surgindo diversas formas de socialização e diversas atividades relacionadas ao descanso e à busca do bem-estar pessoal. Por isso, é necessário aprofundar-se na oferta de propostas de lazer e tempos livres que respondam às atuais necessidades de mudança que se colocam entre os jovens: que tipo de atividades são praticadas durante a semana compatíveis com a vida acadêmica, em que tempo livre é investido durante fins de semana e feriados ou quais as preferências de estudantes de diferentes carreiras universitárias.

\section{METODOLOGÍA}

\section{Diseño de investigación}

Para atingir o objetivo enunciado na seção introdutória, foi proposta uma revisão da literatura, com a qual se pretende realizar uma tarefa sistemática e aprofundada sobre uma área (Bisquerra, 2011, 2016). Através deste trabalho será possível construir um conjunto sólido de conhecimentos oportunos que abordem a problemática da relação entre o lazer dos estudantes universitários, a sua dedicação e envolvimento de acordo com as suas preferências e os fatores socioeducativos, de bemestar e de melhoria da qualidade. de vida de acordo com cada indivíduo.

As revisões bibliográficas fazem parte da pesquisa secundária (Cea D’Ancona, 1996; Pacios, 2013), pois são realizadas por meio de estudos anteriores, evidenciando conhecimentos sobre um 
campo específico e ampliando ideias em torno do objeto de estudo. Além disso, este tipo de estudos e pesquisas têm fases de execução bem diferenciadas (Bernárdez-Gómez et al., 2020): estabelecer os critérios que serão utilizados na busca, coleta e análise preliminar dos documentos a fim de selecionar aqueles que atendam os critérios estabelecidos e, em seguida, a análise final dos textos.

\section{Muestra}

A seleção dos documentos para a realização da análise é uma tarefa que requer uma série de critérios que conferem rigor ao estudo. Para isso, determinou-se que a primeira busca de documentos deveria ser realizada nas bases de dados que proporcionam maior atividade na área educacional: Web of Science, Scopus, Eric e Dialnet. Uma vez introduzidos, os termos que os assinantes mais frequentemente entendem no estudo deste tema educacional foram usados como descritores: \# lazer universitário, \# atividades de lazer juvenis, \# práticas de tempos livres dos alunos. Da mesma forma, a busca foi realizada com os descritores correspondentes na língua inglesa.

Realizada a busca de textos nas bases de dados citadas, emergiu um total de 69 referências com os filtros iniciais que corresponderam aos critérios de inclusão / exclusão: textos em espanhol e inglês e publicados nos últimos cinco anos (2016 -2021). Uma vez verificado que os documentos estavam em acesso aberto e vinculados ao objeto de estudo, resultou a amostra para a análise de 22 artigos.

\section{RESULTADOS Y DISCUSIÓN}

A prática do lazer é muito antiga, mas não é até o final da Segunda Guerra Mundial quando, antagonicamente, devido à grande destruição e ao conseqüente esforço de trabalho para a reconstrução, o lazer é apreciado como algo necessário para o descanso e recuperação., físico e psicológico, e tão digno quanto o próprio trabalho.

Mas do ponto de vista investigativo e sociológico, embora trabalhos teóricos sobre o lazer já tenham surgido a partir dessa época, não será senão quase na segunda metade do século XX que tais estudos começarão a proliferar; e no que diz respeito à pesquisa aplicada, é na segunda metade daquele século, e atualmente, quando muitos pesquisadores já focam seu interesse no lazer, eu o concebo como um bem alcançado pela nossa sociedade e necessário para um bom equilíbrio pessoal. Vários autores tentam explorar o conceito de lazer a partir de uma base empírica (López, Esteve \& San Martín, 1999). Em sua pesquisa, concluem que sob o significado de lazer, dois aspectos claramente desiguais parecem estar subjacentes: por um lado, tudo o que se relaciona com relaxamento, passividade, descanso ou, simplesmente, não fazer nada; de outro, o conjunto de atividades que se relacionam a fazer algo, devido à importância que essas ações têm para o próprio indivíduo. Eles tornam visível a relevância da interação social como variável na prática do lazer. Por outro lado, os pesquisadores enfrentam vários desafios na concepção de escalas psicométricas para medir o significado do lazer. Incluem a necessidade de construir uma concepção com indicadores que permitam avaliar o alcance e a dimensão de um determinado comportamento de lazer e seu 
significado para o sujeito (Schulz \& Watkins, 2007). Os dois autores realizam um estudo que descreve o desenvolvimento do descritivo dos significados do lazer, como uma escala multidimensional para mensurar quatro modos qualitativamente diferentes de vivenciar o significado do lazer: a passagem do tempo, escapar de pressões, exercitar a escolha e alcançar a realização.

Embora muitos dos dados referentes ao lazer estejam relacionados aos estudos sociológicos, em um nível geral sobre os jovens (Comas et al., 2003; Instituto de la Juventud, 2004a, 2004b, 2006, 2007; Rodríguez \& Agulló, 1999), em o Quanto aos últimos estudos empíricos revisados sobre atividades de lazer em estudantes universitários, não só são analisadas as características das atividades de lazer e sua frequência de escolha pelos jovens, mas também abordam outros aspectos, além das descrições adequadas. Como é o caso de variáveis como fatores de lazer inclusivos (Izuzquiza, 2010; Madariaga et al., 2010; Schulz \& Watkins, 2007; Vicente, 2015), o consumo de práticas culturais (Cuenca 2000, 2014; Rodríguez \& Agulló, 1999 ), consumo de álcool e drogas (Megías E. et al., 2005; Expósito et al, 2009), diferenças nas preferências de lazer em carreiras técnicas e não técnicas (Lemp \& Behn, 2008), percepção modificada por gênero (Cuenca, 1999 ; Sánchez-Herrero, 2008), a experiência de lazer (Cuenca 2014; Olubor \& Osunde, 2007), transculturalidade e incorporação do lazer como estilo de vida (Cuenca, 2006; Echazarreta et al., 2016; Gokturk, 2009; Hiu - Lun Tsai \& Coleman, 2007; Mirsafian, 2016), crenças e atribuições de autoeficácia (Walker e Wang, 2008; Wise, 2009), lazer como meio para atingir objetivos organizados, a fim de em si (Bedini et al., 2011 ; Patry et al., 2007), estilos de aprendizagem (Alonso et al., 2012; García-Cué \& Santizo, 2010; Ló Pez Alonso, 2011). Sua finalidade está relacionada à possibilidade de intervir por meio dessas correlações existentes em costumes, atitudes, etc., de jovens universitários para melhorar sua qualidade de vida.

De alguma forma, cada estudo valoriza as atividades de lazer e fortalece as teorias de que, em nossa sociedade, o lazer além de ter uma função evasiva, é um momento onde os jovens originam relações, trocas de normas, comportamentos, valores ( Instituto de Tecnologias Educacionais, 2007), e destacar o potencial das experiências de lazer como um local ideal para transmitir valores para uma convivência saudável (Castañeda et al., 2018; Valdemoros, 2008), além de ser um estilo de crescimento pessoal e desenvolvimento (García - Cué \& Santizo, 2010; Porter et al., 2010; Schulz \& Watkins, 2007), e ainda explicam como certas atividades de lazer realizadas têm um impacto direto na aprendizagem (Caladine, 2008; Patry et al. , 2007). Em seu livro, Caladine (2008 p.4-6), explica como as experiências de lazer online, por meio de seus conteúdos multimídia, e os tipos de interação que atualmente prevalecem nas experiências de lazer, são referidas como uma metodologia facilitadora e motivadora de experiências efetivas de aprendizagem desta maneira. $\mathrm{Na}$ mesma linha, encontram-se os trabalhos desenvolvidos na Universidade de Jaén por Gómez \& Tapia (2011) ou na Universidade Pompeu Fabra por Carratalá (2015), que propõem o uso das principais redes sociais utilizadas pelos universitários espanhóis em geral. como atividade de lazer, como ferramenta pedagógica relacionada a um potencial de produtividade na aprendizagem neste meio, facilitada, entre outros componentes, pela característica de um canal interativo, e acabam concordando que é possível utilizar essas ferramentas como complemento substancial para a 
aprendizagem presencial e colaborativa em sala de aula.

Da mesma forma, há estudos que avaliam a influência de determinadas intervenções por meio de programas de lazer que otimizam as interações, no nível pessoal e com o meio cultural, neste caso. É Kim (2010) quem utiliza um programa lúdico, conheça sua cidade, como fórmula para os participantes descobrirem novas possibilidades sobre a cultura. A participação voluntária neste tipo de lazer recreativo induz uma aprendizagem motivadora, que inspira confiança e sentimento de realização, proporcionando aos jovens a oportunidade de perceber a cultura e não apenas de teorizar sobre ela; ajuda os participantes a adquirir novas habilidades de aprendizagem e descobrir seu potencial, e melhora o desenvolvimento do pensamento flexível e criativo; por outro lado, a utilização de novas tecnologias, muito atrativas para os participantes; câmeras de vídeo ou telefones celulares facilitam uma atitude muito positiva e altamente motivada.

Outro estudo interessante é o de Danielle A. Patry et al. (2007) que propõem que uma orientação acadêmica autodeterminada facilita uma abordagem mais adaptativa para 0 gerenciamento da regulação do lazer, o que resulta em uma menor quantidade de estresse e maior bem-estar emocional.

Os professores Rodríguez e Agulló (1999) realizaram um estudo com alunos da Universidade de Oviedo focalizando seu interesse em conhecer a natureza das práticas culturais de lazer dos universitários, com o intuito de poder realizar uma tipologia de práticas e consumos culturais básicos, que permitirão uma programação de lazer cultural mais coerente e satisfatória para esses alunos. Nele, conclui-se que o universitário conformava um estilo de vida próprio e diferente dos demais jovens, por consumir mais atividades culturais.

Mais perto dos nossos dias, os perfis das actividades de lazer têm variado e várias fontes associam-nas a uma forte componente social e à noite (Instituto de la Juventud, 2007). Outro estudo, conduzido com uma grande amostra de estudantes universitários de Hong Kong e Austrália, sugere que os ambientes sociais e culturais são fatores importantes que influenciam o interesse na participação ativa e na seleção de entretenimento para os jovens (Hiu-Lun Tsai \& Coleman, 2007).

Exposito et al. (2009) constatam que durante os dias de trabalho os estudantes universitários ocupam principalmente seu tempo livre em atividades de lazer passivo; o que mais fazem nos finais de semana são atividades sociais e lúdicas, como nos curtos períodos de férias como Páscoa, Natal e fins de semana prolongados, deixando as atividades físico-esportivas para o período mais longo que é o verão. Esses resultados corroboram os encontrados em estudos anteriores de Ruiz-Juan (2001, citado em Expósito et al., 2009). Assim, este autor também confirma que os jovens realizam suas atividades principais, nos finais de semana, em ambientes onde o álcool é frequente; Têm larga experiência no seu consumo e um perfil de intensidade muito variado. $O$ aumento do consumo nos finais de semana está associado a uma maior prevalência e intensidade de consumo e ressacas durante a semana. 
No México, Lemp \& Behn (2008) investigam que os estudantes universitários em carreiras não técnicas passam mais tempo estudando do que os estudantes em carreiras técnicas, descobrindo que estes últimos compartilham seu tempo acadêmico com o tempo de trabalho remunerado. Outro achado significativo é que os homens têm maior percepção de disponibilidade de tempo livre do que as mulheres e que os que mais bebem são os que percebem que têm mais tempo.

Por outro lado, deve-se destacar que a experiência de lazer e seu construto não é transcultural. Gokturk (2009) destaca em seu estudo a experiência de lazer, medida por meio da bateria LEBYA (Barnett, 2005; citado em Gokturk, 2009), destinada a jovens estudantes matriculados em aulas de inglês em uma universidade da Turquia, e que por isso cresceram em culturas diferentes da dos EUA, que embora para certos fatores, como tédio, desafio, luto e conhecimento, as conclusões fossem muito semelhantes às encontradas para amostras de estudantes norteamericanos, houve diferenças significativas relacionadas às subescalas de desafio, que não se correlacionou com conhecimento e tédio, com luto, e estes não variaram com o gênero. De tudo isso, segue-se que a pesquisa transcultural precisa esclarecer melhor essas diferenças atribuídas à cultura ou etnia, na experiência do lazer.

E nessa linha de diferentes percepções sobre a experiência de lazer, vale citar a metanálise realizada por Sánchez-Herrero (2008), que realizou uma revisão teórico-empírica sobre o tempo livre e o lazer nas mulheres a partir de três aspectos básicos: a quantidade de tempo livre e suas características e condições, a qualidade do tempo livre, o equilíbrio entre vida e trabalho, conceitos de tempo livre ... e conteúdo do tempo livre. A sua conclusão mais significativa foi que as características biológicas, económicas, sociais e laborais de grande parte das mulheres tornam a quantidade, o conteúdo e a qualidade do lazer e do tempo livre diferentes e, frequentemente, piores que os dos homens. Este estudo é importante, visto que a população estudada possuía um número muito grande de mulheres.

Olubor \& Osunde (2007) realizaram um estudo em várias universidades nigerianas, procurando conhecer o tempo semanal destinado ao estudo e às tarefas pessoais de estudantes universitários nigerianos. Um dos resultados mais marcantes foi que esses alunos não incorporaram a cultura do lazer, como é interpretada no Ocidente, em seu estilo de vida e, portanto, não sabiam como organizar seu tempo livre. A maioria dos estudantes universitários passava vinte e seis horas por semana estudando, e uma minoria dez horas ou menos. A sua conclusão foi que existem outras actividades que competem com o tempo de estudo, que são desconhecidas e que têm uma implicação negativa no seu compromisso com os estudos, pelo que a própria Universidade foi incorporando nos seus lobbies, como mecanismo de resolução das necessidades recreativas dos alunos, suas atividades preferidas, como música e jogos de tabuleiro, embora não tivessem recursos suficientes para oferecer oportunidades equivalentes em cada universidade.

Andrijašević et al. (2005) relacionaram a percepção do estado de saúde de estudantes universitários com suas atividades esportivas e de lazer. No trabalho realizado com estudantes universitários, neste caso de diferentes Faculdades da Universidade de Split, constatam que quem 
pratica atividades desportivas, na sua maioria homens, tem menos desconforto físico e que as mulheres que têm mais tempo livre são percebidas como mais saudável. Em última análise, eles correlacionam os desconfortos psicossomáticos descritos no estudo com estilos de vida inativos ou sedentários. Outro estudo (Patry et al., 2007), também realizado com estudantes universitários, sugere a evidência de que as atividades de lazer podem ser utilizadas como meio de enfrentar o estresse, mas a forma como o lazer é utilizado, como forma eficaz de regular tarefas difíceis, ainda não está claro.

Wise (2009), através de um estudo realizado na Universidade de Minnesota, utiliza três atividades exigentes de lazer ativo para predizer a autoeficácia de estudantes universitários, chegando às seguintes conclusões: (a) que características pessoais, atributos pessoais, quanto ao desempenho de um esforço físico, em uma atividade de lazer e em um determinado contexto, preveem autoeficácia frente àquela situação; (b) que as crenças de autoeficácia em um determinado contexto, compreendidas e refletidas pela pessoa, podem contribuir para uma nova visão das crenças e reforçar a eficácia em outra nova situação; e (c), que ao aumentar a frequência com que os universitários participam fisicamente de atividades ativas de lazer, se fortalece a autoeficácia para a superação de restrições, e os alunos adquirem novos atributos pessoais, vistos como transpor barreiras e se esforçar. De certa forma, a pesquisa realizada por Hiu-Lun Tsai \& Coleman (2007), chega a propor algo semelhante, uma vez que os resultados deste estudo comparativo entre estudantes universitários de Hong Kong e estudantes australianos mostram que, mesmo levando em consideração as diferenças de Nos dois grupos, os participantes mais ativos têm maior predileção por recreação ativa e têm menor percepção quanto às restrições de tempo livre. Esses mesmos autores (Hiu - Lun Tsai \& Coleman, 2009), em amostras de estudantes universitários dos mesmos países mencionados acima, concluem que a eficácia é uma força importante no interesse da participação dos alunos, e que aqueles com melhores técnicas e condições físicas tendem para manter níveis mais altos de interesse na recreação ativa.

Outro estudo analisado é o realizado por García - Cué \& Santizo (2010), que relaciona a ocupação do tempo livre e estilos de aprendizagem. Embora não se refira a universitários e sim a egressos, é interessante, pois é o único que se encontra relacionado a ela de alguma forma, ainda mais quando se conclui que as pessoas ocupam seu tempo livre influenciadas por estilos de aprendizagem. Dessa forma, os altos valores do estilo ativo foram relacionados aos fãs de videogame; altos valores no estilo reflexivo, com ouvir música e praticar esportes; altos valores no estilo teórico, com ouvir música, ir ao cinema e ouvir rádio, e altos valores no estilo pragmático, com ir às compras.

Caride et al. (2018) em sua pesquisa sobre a relação entre a leitura, entendida como atividade passiva de lazer, e estudantes de ciências da educação na Universidade de Santiago de Compostela, conclui que se nos concentrarmos na ligação entre o hábito de leitura e as práticas de lazer, os dados mostram que as pessoas que gostam de ler tenham uma visão mais diversificada do lazer e valorizem mais positivamente o seu tempo livre. 
O último estudo analisado por Cuberos et al. (2016) sobre a adesão da dieta mediterrânea em universitários e sua relação com o lazer sedentário, conclui-se que dada a baixa porcentagem de universitários que seguem uma dieta de ótima qualidade e sua correspondência com o uso de videogames, é de importância vital a criação de programas de intervenção que otimizem a alimentação deste setor e reduzam o tempo de lazer sedentário.

\section{CONCLUSIONES}

Da análise efetuada podem tirar-se várias conclusões, a primeira delas é que as atividades de lazer ativo propostas no ambiente universitário com curta duração (Montes et al., 2015) favorecem a própria prática dos jovens universitários e implicam um melhor. qualidade de vida e bem-estar social. Da mesma forma, os alunos que apresentam melhores condições físicas e técnicas tendem a demonstrar maior interesse pela prática do lazer ativo. Por outro lado, os alunos que praticam atividades físico-desportivas e seguem uma dieta mediterrânea de qualidade apresentam menos desconforto físico e são mais saudáveis. No que se refere à experiência de lazer entre homens e mulheres, as conclusões obtidas quanto às características biológicas, econômicas, sociais e laborais de grande parte das mulheres tornam a quantidade, o conteúdo e a qualidade do lazer e do tempo livre diferentes e muitas vezes menos positivos que os dos homens. No entanto, a instituição acadêmica ainda desempenha um papel fundamental, a responsabilidade educacional não se limita a ela, mas a superação dos estereótipos de gênero exige o compromisso inevitável de vários agentes como as famílias, a mídia, as administrações locais ou os serviços de lazer. A conformação dos interesses ou gostos sobre os quais se ratificam as experiências de lazer desportivo e as oportunidades que os jovens têm de as vivenciar, transcende amplamente os contextos escolares, sendo determinada nos diversos cenários em que passa o seu dia a dia, sendo o fosso de género um fator essencial a ser levado em consideração (Fraguela-Vale et al. 2020). E, nesse sentido, a experiência de lazer também não é transcultural.

Conforme coletado em seu trabalho Elche et al. (2019), o centro educacional é fundamental para reforçar as atividades de lazer. As universidades oferecem aos seus alunos oportunidades importantes de tempos livres, disponibilizando infraestruturas, equipamentos e atividades como complemento da formação dos alunos. Nessa mesma linha de conclusões, conclui-se que será positivo tomar medidas para esse fim, a fim de determinar os meios para a realização de tais atividades, tais como as sugeridas pelos próprios alunos; cinema de debate, espaços de

recreação mais delineada do ponto de vista lúdico, esportes intra e inter-alunos ou encontros de férias para promover a sociabilidade, principalmente nos primeiros anos da vida acadêmica universitária (Pascucci, 2015). Da mesma forma, Caride et al. (2018) compartilham a visão de que o corpo docente deveria assumir um papel maior na promoção do hábito da leitura de seus alunos, cabendo à Universidade ativar políticas institucionais que favoreçam os espaços e promovam novas oportunidades para alcançar uma maior articulação entre a leitura e os tempos de lazer; incorporar na formação dos futuros profissionais da educação competências que thes dêem um papel ativo no trabalho da leitura. 
É importante mencionar que a pedagogia do lazer e dos tempos livres é uma disciplina que inicia sua consolidação a partir do momento em que as sociedades alcançam um mínimo de qualidade de vida. Em 1968, Dumazedier (Puig e Trilla. 2000) aponta que o lazer é um espaço, uma experiência, um recurso, uma fonte de saúde, um direito humano e um tempo característico da sociedade atual. E por isso é necessário definir algumas ações educativas que permitam contribuir para a expansão e consolidação do lazer (Sandoval, 2017).

\section{REFERENCIAS}

Andrijašević, M., Paušić, J., Bavčević, T., \& Ciliga, D. (2005). Participation in leisure activities and selfperception of health in the students of the University of Split. Kinesiology, 37(1), 21-31.

Alonso, A. I. L., López-Aguado, M., González-Millán, I., \& Martínez, M. E. F. (2012). El ocio y los enfoques de aprendizaje en estudiantes universitarios. Revista de investigación Educativa, 30(1), 53-70. https://doi.org/10.6018/rie.30.1.111581

Bedini, L. A., Gladwell, N. J., Dudley, W. N., \& Clancy, E. J. (2011). Mediation analysis of leisure, perceived stress, and quality of life in informal caregivers. Journal of Leisure Research, 43(2), 153-175.

Belmonte Almagro, M. L., \& Bernárdez-Gómez, A. (2021). Evaluation of Self-Concept in the Project for People with Intellectual Disabilities: "We Are All Campus". Journal of Intelligence, 9(4), 50. https://doi.org/10.3390/jintelligence9040050

Bernárdez-Gómez, A., Marafante, G., Da Silva, L. (2020). Perspectivas teóricas sobre o engajamento agêntico. Brazilian Journal of Development, Curitiba, 6(10), 75648-75661. https://doi.org/10.34117/bjdv6n10-116

Bernárdez-Gómez, A., \& Belmonte, M. L. (2020). Evasão escolar, determinantes, políticas educacionais e itinerários subsequentes. Research, Society and Development, 9(10), e6849109234-e6849109234. https://doi.org/10.33448/rsd-v9i10.9234

Bernárdez-Gómez, A., González Barea, E.M. \& Rodríguez Entrena, M.J. (2021). Educational Reengagement Programs and Measures in the Region of Murcia. An Approach to their Understanding. International Journal of Sociology of Education, 10(3), 246-270. http://doi.org/10.17583/rise.7516

Bisquerra, R. (2011). Diversidad y escuela inclusiva desde la educación emocional. Diversidad, Calidad y Equidad Educativas, 1-9.

Bisquerra, R. (2016). Metodología de la investigación educativa. La Muralla.

Caladine, R. (2008). Enhancing e-learning with media-rich content and interactions. Information Science Publishing. https://doi.org/10.4018/978-1-59904-732-4

Caride, J. A., Caballo, M. B., \& Gradaílle, R. (2018). Reading in leisure time: students, future professionals of education, as readers Leer en tiempos de ocio: los estudiantes, futuros profesionales de la educación, como sujetos lectores. Ocnos, 17(3), 7-18. https://doi.org/10.18239/ocnos 2018.17.3.1707

Carratalá, A. (2015). El empleo de herramientas de debate asíncronas con alumnos de periodismo: entre el foro virtual y la red social. In Periodismo actual y futuro: investigación, docencia e innovación: Actas del XX Congreso Internacional de la Sociedad Española de Periodística 
(pp. 1181-1204). Universitat Pompeu Fabra.

Castañeda Sáenz, K. A., Sevilla Morocho, L. E., Calero Morales, S., Romero Frómeta, E., Torres Ramírez, A., \& Romero Gómez, Y. (2018). Estado anímico en el tiempo libre de estudiantes que inician y culminan la universidad. Revista Cubana de Investigaciones Biomédicas, 37(2), 27-38. https://doi.org/10.25176/rfmh.v21i3.4021

Cea D’Ancona, M. A. (1996). Metodología Cuantitativa. Estrategias y técnicas de investigación social. Síntesis.

Comas, D., Aguinaga, J., Andrés, F., Espinosa, A., \& Ochaita, E. (2003). Jóvenes y estilos de vida: valores y riesgos en los jóvenes urbanos. Fundación de Ayuda contra la Drogadicción. https://doi.org/10.2307/40184663

Cuberos, R. C., Sánchez, M. C., Molina, J. J. M., Garcés, T. E., Ortega, F. Z., \& Manrique, M. L. (2016). Adhesión a la dieta mediterránea en estudiantes universitarios y su relación con los hábitos de ocio digital. Nutrición hospitalaria: Organo oficial de la Sociedad española de nutrición parenteral y enteral, 33(2), 405-410. https://doi.org/10.20960/nh.124

Cuenca, M. (1999). Hacia la equiparación de oportunidades mediante la Educación de Ocio. Universidad de Deusto.

Cuenca, M. (2000). Ocio humanista: dimensiones y manifestaciones actuales del ocio. Universidad de Deusto.

Cuenca, M. (2006). Aproximación multidisciplinar a los estudios de ocio. Universidad de Deusto.

Cuenca, M. (2014). APROXIMAÇÃO DO ÓCIO VALIOSO. Revista brasileira de estudos do lazer, $1(1), 21-41$

Cuenca, M. (2014). Ocio valioso. Universidad de Deusto.

Echazarreta, R. R., Maulini, C., Migliorati, M., \& Isidori, E. (2016). Adolescencia y estilo de vida. Estudio del ocio de los escolares de la provincia de Roma. Pedagogía social: revista interuniversitaria, (28), 127-139.

Elche, M., Sánchez-García, S., \& Yubero, S. (2019). Lectura, ocio y rendimiento académico en estudiantes universitarios del área socioeducativa.

https://doi.org/10.5944/educxx1.21548

Expósito, J., García, L., Sanhueza, C. \& Angulo, M. T. (2009). Análisis de las actividades de ocio en estudiantes de primer curso de la Facultad de Educación: Relación con el alcohol. Revista Complutense de Educación, 20(1), 165-192.

Fraguela-Vale, R., Varela Garrote, L., \& Varela-Crespo, L. (2020). Perfiles de ocio deportivo en jóvenes españoles (15-20 años): un análisis de género. Retos: nuevas tendencias en educación física, deporte y recreación, 37, 419-426.

García-Cué, J. L. \& Santizo, J. A. (2010). Análisis de la relación entre la gestión del tiempo libre, el ocio y los estilos de aprendizaje. Revista estilos de aprendizaje, 5(5), 2-24.

Gokturk, D. (2009). A study of the leisure experience of turkish university students. College Student Journal, 43(2), 303-324.

Gómez, B. \& Tapia, A. (2011). Facebook y Tuenti: de plataforma de ocio a herramienta e-learning. Prisma Social: revista de ciencias sociales, 6, 238-260. https://doi.org/10.4185/rlcs-66-2011- 


\section{5-079-100-en}

Hiu-Lun Tsai, E. \& Coleman, D. J. (2007). Preferences for active recreation and perceived constraints to regular active recreation participation: a cross-cultural study of Hong Kong and Australian University students. Leisure/Loisir: Journal of the Canadian Association for Leisure Studies, 31(1), 155-189. https://doi.org/10.1080/14927713.2007.9651377

Instituto de la Juventud. (2004a). Informe Juventud en España 2004. Resultados marginales. Estudio INJUVE EJ089. Madrid: INJUVE. Disponible en http://www.injuve.mtas.es

Instituto de la Juventud. (2004b). Sondeo de opinión y situación de la gente joven. Ocio y tiempo libre, noche y fin de semana, consumo de alcohol, inmigración. Segunda encuesta 2004. Avance de resultados. Estudio INJUVE EJ100. INJUVE. http://www.injuve.migualdad.es/

Instituto de la Juventud. (2006). Sondeo de opinión y situación de la gente joven. Percepción generacional, valores y actitudes, calidad de vida y felicidad. Segunda encuesta 2006. Tabla de resultados. Estudio INJUVE EJ113. Madrid: INJUVE. http://www.injuve.migualdad.es/

Instituto de la Juventud. (2007). Sondeo de opinión y situación de la gente joven. Uso de tecnologías, ocio y tiempo libre. Segunda encuesta 2007. Estudio INJUVE EJ124. INJUVE. http://www.injuve.migualdad.es/.

Izuzquiza Gasset, D. (2004). El ocio en las personas con Síndrome de Down (Doctoral dissertation, Universidad Complutense de Madrid, Servicio de Publicaciones).

Kim, S. H. (2010). An exploration of the applicability of a recreational Orientierungslauf (orienteering) programme for urban cultural experience activities. Journal of Hospitality, Leisure, Sports and Tourism Education (Pre-2012), 9(2), 130. https://doi.org/10.3794/johlste.92.279

Lemp, M. \& Behn, V. (2008). Utilización del tiempo libre de estudiantes del área de salud de la institución educacional Santo Tomas, Talca. Leisure activities of students from the health area in Santo Tomas Educational Institute, Talca. Ciencia Enfermera, 14(1), 53-62.

López, A. E., Esteve, R. \& San Martín, J. (1999). Dimensionalización del constructo de ocio en universitarios. Psicothema, 11(1), 113-124

López Alonso, A. I. (2011). Enfoques de aprendizaje de los alumnos universitarios. Interrelación con hábitos de ocio, engagement y percepción de bienestar. Propuesta de un modelo estructural= Approaches of learning on University students. Interrelationship with leisure habits, engagement and well-being perception. Proposal of a structural model. Tesis Doctoral. Universidad de León. https://doi.org/10.18002/10612/5918

Megías, E., Rodríguez, E., Megías, I., \& Navarro, J. (2005). La percepción social de los problemas de drogas en España, 2004. Fundación de ayuda contra la drogadicción.

Mirsafian, H. (2016). Perceived constraints to physical activity and sport: a cross-cultural study between Iranian and Hungarian university students. World Leisure Journal, 58(3), 193-206. https://doi.org/10.1080/16078055.2016.1143390

Montes, M. E. G., Juan, F. R., \& López, M. G. (2005). Cómo ocupan los universitarios almerienses su tiempo libre. Lecturas: Educación física y deportes, (83), 10.

Olubor, R. \& Osunde, U. (2007). Analysis of personal study time and leisure among university undergraduates in southern Nigeria. College Student Journal, 41(2), 352-365. 
Pacios, A. (2013). Técnicas de búsqueda y uso de la información. Editorial Universitaria Ramón Areces.

Pascucci, M. (2015). Los jóvenes universitarios y el ocio. European Scientific Journal.

Patry, D. A., Blanchard, C. M., \& Mask, L. (2007). Measuring university students' regulatory leisure coping styles: Planned breathers or avoidance?. Leisure Sciences, 29(3), 247-265. https://doi.org/10.1080/01490400701257963

Porter, H., Iwasaki, Y., \& Shank, J. (2010). Conceptualizing meaning-making through leisure experiences. Loisir Et Société/Society and Leisure, 33(2), 167-194. https://doi.org/10.1080/07053436.2010.10707808

Rodríguez, J. \& Agulló, E. (1999). Estilos de vida, cultura, ocio y tiempo libre de los estudiantes universitarios. Psicothema, 11(2), 247-259.

Sánchez-Herrero, S. A. (2008). La importancia de la perspectiva de género en la psicología del ocio. Anales de Psicología, 24(1), 64-76.

Sandoval, N. (2017). Diagnóstico acerca del uso del ocio y el tiempo libre entre los estudiantes de la Universidad Nacional Experimental del Táchira. https://doi.org/10.7179/psri 2017.30.12

Schulz, J. \& Watkins, M. (2007). The development of the leisure meanings inventory. Journal of Leisure Research, 39(3), 477-497.

Trillo Alonso, F., Gañete, A. P., \& Bernárdez-Gómez, A. (2019). Juan, a Child with Asperger's Syndrome: A Case Study of a Good Practice of Educational Inclusion Through Cooperative Learning. Revista Brasileira de Educação Especial, 25, 85-100. https://doi.org/10.1590/S141365382519000100006

Valdemoros, M. A., Ponce de León, A., Sanz, E. \& Ramos, R. (2008). Valores para la convivencia en los adolescentes desde las experiencias de ocio físico-deportivo. In IV Congreso Internacional y XXV Nacional de Educación Física. Universidad de Córdoba

Vicente, P. (2015). El ocio en las personas con discapacidad intelectual, un acercamiento al ocio inclusivo. (Doctoral dissertation, Universidad de Murcia).

Walker, G. J., \& Wang, X. (2008). A cross-cultural comparison of Canadian and Mainland Chinese university students' leisure motivations. Leisure Sciences, 30(3), 179-197. https://doi.org/10.1080/01490400802014420

Wise, J. (2009). Using the knowledge-and-appraisal personality architecture to predict physically active leisure self-efficacy in university students. Journal of Applied Social Psychology, 39(8), 1913-1927. 